\title{
Влияние дегидрирования графана на его механические и электронные свойства
}

\author{
(С Л.А. Опенов, А.И. Подливаев \\ Национальный исследовательский ядерный университет „МИФИ“, \\ 115409 Москва, Россия \\ ๑ E-mail: AIPodlivayev@mephi.ru
}

(Получена 25 апреля 2017 г. Принята к печати 1 июня 2017 г.)

\begin{abstract}
В рамках неортогональной модели сильной связи исследовано влияние десорбции водорода на механические характеристики и электронную структуру кресельной конформации графана. Показано, что механическая жесткость и коэффициент Пуассона немонотонно зависят от содержания водорода и минимальны при концентрации водородных вакансий $\sim 50$ и $\sim 30 \%$ соответственно. Характеристические пики плотности фононных состояний при десорбции водорода быстро уменьшаются. На начальной стадии десорбции в запрещенной зоне возникают локальные уровни энергии. По мере увеличения числа водородных вакансий эти уровни формируют примесную зону, в которой находится уровень Ферми.
\end{abstract}

DOI: 10.21883/FTP.2018.06.45925.8622

\section{1. Введение}

Графен - монослой графита - представляет собой квазидвумерную наноструктуру с гексагональной решеткой [1]. Интерес к этому материалу обусловлен его уникальными физическими свойствами. Так, аномально высокая подвижность носителей заряда делает графен перспективным для использования в электронике [2]. Макроскопический лист графена является бесщелевым полупроводником, однако для практических приложений из него можно приготовить образцы с конечной шириной запрещенной зоны $E_{g}$. Например, оказывается возможным изменять величину $E_{g}$, разрезая лист графена на ленты различной ширины [3-5]. Более радикальным и удобным способом формирования в электронном спектре графена запрещенной зоны является его гидрирование. При полном двустороннем гидрировании образуется графан (graphane) - сначала предсказанный в работе [6], а затем и синтезированный (см. работу [7]) квазидвумерный углеводородный материал, являющийся широкозонным диэлектриком. В литературе обсуждалось много модификаций графана, отличающихся друг от друга расположением атомов водорода $[6,8,9]$. Наиболее устойчивой модификацией считается кресельная (chair) конформация, в которой атомы водорода, адсорбированные на соседних атомах углерода, расположены по разные стороны от плоскости монослоя. Именно эта конформация, привлекающая к себе внимание большинства исследователей, и рассматривается в настоящей работе. Ее отличительной особенностью является (как и у графена) изотропия упругих характеристик: модуль Юнга и коэффициент Пуассона практически не зависят от направления растяжения кристалла, тогда как в некоторых других конформациях имеет место сильная угловая зависимость этих характеристик, вплоть до смены знака у коэффициента Пуассона [10].

Частично дегидрированный графан может обладать свойствами, полезными для некоторых технических при- ложений. Например, магнетизм водородных вакансий в графане $[8,11-13]$ позволяет рассматривать графан с небольшой концентрацией таких вакансий как возможный элемент памяти ЭВМ. Устойчивость и возможность спонтанной регенерации атомарно резкой границы раздела графен/графан при термическом разупорядочении [14] дает надежду на создание термостойких электронных приборов на основе частично гидрированного графена (частично дегидрированного графана). Одностороннее гидрирование графена полосами адсорбированного водорода также рассматривалось в контексте создания наноэлектронных приборов [5,15].

Гидрирование графена (дегидрирование графана) сильно влияет на электронные и механические характеристики этих материалов [16-19]. Так, в работе [16] была обнаружена немонотонная зависимость коэффициента Пуассона графана от содержания в нем водорода (коэффициент Пуассона оказался минимальным при половинном дегидрировании графана). Производные графена с различным количеством адсорбированных на нем атомов водорода интересны не столько для электронных приложений (в этом случае чаще требуются упорядоченные структуры), сколько для создания элементов водородных аккумуляторов. Предпосылкой к этому является высокая удельная поверхность графена и графана, а также их способность как к адсорбции водорода (графен), так и к его термической десорбции (графан) [20].

Для электронных приложений частично дегидрированного графана представляют интерес результаты работы [21]. В этой работе показано, что в зависимости от конфигурации и концентрации периодически расположенных атомов водорода запрещенная зона может иметь различную ширину или вовсе отсутствовать. Возможно также появление новых пиков плотности состояний.

Цель настоящей работы - исследование влияния хаотически расположенных водородных вакансий, образующихся при десорбции водорода, на фононный спектр, 
упругие модули и плотность электронных состояний кресельной конфигурации графана. Наш интерес обусловлен отчасти перспективой экспериментальной регистрации и диагностики этой конформации с нестехиометрическим содержанием водорода.

На примере графена известно, что теоретические значения механических характеристик квазидвумерных материалов (модуль Юнга и коэффициент Пуассона) чрезвычайно чувствительны к методу расчета и деталям вычислительной процедуры (см. работу [22] и ссылки в ней). В отсутствие надежной экспериментальной информации об упругих модулях графана представляется целесообразным их расчет в рамках самых различных подходов. В настоящей работе для этих целей использован неортогональный метод сильной связи.

\section{2. Методы расчета}

Мы моделировали кресельную конформацию графана прямоугольной 320-атомной сверхъячейкой $\mathrm{C}_{160} \mathrm{H}_{160}$ (рис. 1), состоящей из 80 примитивных элементарных ячеек $\mathrm{C}_{2} \mathrm{H}_{2}$. Десорбции водорода из графана соответствует уменьшение числа атомов водорода в этой сверхъячейке. Мы рассматривали сверхъячейки $\mathrm{C}_{160} \mathrm{H}_{x}$ с $0<x<160$, которые получали, удаляя случайным образом атомы водорода из исходной сверхъячейки с $x=160$ ( $x=0$ отвечает графену). Использовались периодические граничные условия в двух планарных направлениях $(X, Y)$ и свободные в поперечном направлении $(Z)$. Периоды каждой сверхъячейки определялись из условия минимума ее энергии после релаксации по координатам всех атомов.

Межатомные взаимодействия $\mathrm{C}-\mathrm{C}, \mathrm{C}-\mathrm{H}$ и $\mathrm{H}-\mathrm{H}$ описывались в рамках неортогональной модели сильной связи [23], которая в явном виде учитывает вклад всех валентных электронов атомов углерода $\left(2 S, 2 P_{x}, 2 P_{y}\right.$, $\left.2 P_{z}\right)$ и водорода $(1 S)$ в полную энергию. Эта модель уступает по точности первопринципным методам, но гораздо менее требовательна к вычислительным ресурсам и позволяет детально изучить поверхность потенциальной энергии системы из нескольких сотен атомов. Ранее

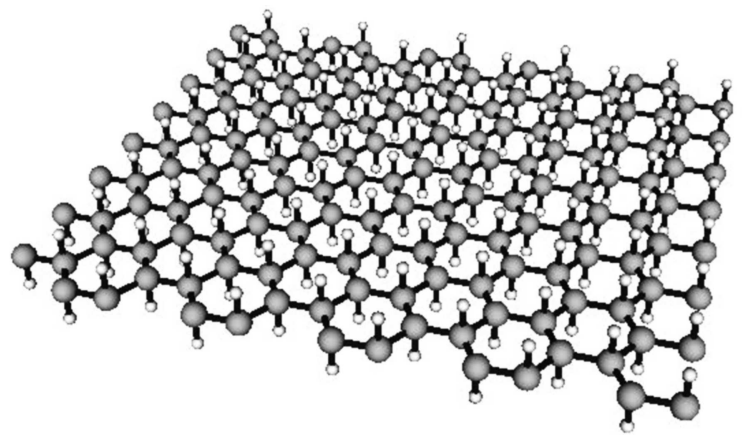

Рис. 1. Сверхъячейка $\mathrm{C}_{160} \mathrm{H}_{160}$. Большие шарики - атомы углерода, маленькие шарики - атомы водорода. мы использовали ее для исследования статических и динамических характеристик различных углеводородных наноструктур, в том числе графана [20], границы раздела графан/графен [14], гидрированных нанотрубок [24], алмазоподобных углеродных нанониток [25] и др.

Для расчета спектров собственных колебаний мы численно диагонализовали соответствующую динамическую матрицу. Эти спектры затем использовались для определения плотности фононных состояний (phonon density of states, PDOS).

\section{3. Результаты и их обсуждение}

Прежде чем приступить к изложению и анализу полученных результатов, найдем энергию $E_{1}$, которая требуется для десорбции одного атома водорода из графана. Используя для ее расчета стандартную формулу

$$
E_{1}=E\left(\mathrm{C}_{160} \mathrm{H}_{159}\right)+E(\mathrm{H})-E\left(\mathrm{C}_{160} \mathrm{H}_{160}\right) \text {, }
$$

где $E\left(\mathrm{C}_{160} \mathrm{H}_{159}\right)$ и $E\left(\mathrm{C}_{160} \mathrm{H}_{160}\right)$ - энергии сверхъячеек $\mathrm{C}_{160} \mathrm{H}_{159}$ и $\mathrm{C}_{160} \mathrm{H}_{160}$ соответственно, $E(\mathrm{H})$ энергия изолированного атома водорода, мы получили $E_{1}=3.65$ эВ. Такая энергия может быть передана атому водорода либо при облучении графана (например, электронами), либо при сильном повышении его температуры, что мы непосредственно наблюдали в ходе моделирования динамики сверхъячейки $\mathrm{C}_{160} \mathrm{H}_{160}$, нагретой до $T \sim 1000 \mathrm{~K}$ (при понижении температуры характерное время десорбции быстро возрастает). Заметим, что величина $E_{1}$ меньше энергии связи графана в расчете на один атом $\left(E_{b}=4.83\right.$ эВ для сверхъячейки $\left.\mathrm{C}_{160} \mathrm{H}_{160}\right)$.

Энергия, необходимая для десорбции каждого последующего атома водорода, уменьшается по мере увеличения числа водородных вакансий. Это видно, например, из того факта, что для десорбции 10\% водорода из сверхъячейки $\mathrm{C}_{160} \mathrm{H}_{160}$ (т.е. для создания в ней 16 водородных вакансий) нужно затратить энергию $E\left(\mathrm{C}_{160} \mathrm{H}_{144}\right)+16 E(\mathrm{H})-E\left(\mathrm{C}_{160} \mathrm{H}_{160}\right)=52.8$ эВ или 3.30 эВ/вакансию $<E_{1}$; для десорбции $50 \%$ водорода энергию 2.67 эВ/вакансию и т.д.

\section{1. Плотность фононных состояний}

В спектрах колебаний всех изученных нами сверхъячеек $\mathrm{C}_{160} \mathrm{H}_{x}$ мнимые частоты отсутствуют, т.е. соответствующие атомные конфигурации являются метастабильными и отвечают локальным минимумам поверхности потенциальной энергии. На рис. 2, $a$ представлена PDOS исходной (без водородных вакансий) сверхъячейки (см. также работу [26]). На ней различаются четыре характерных участка: 1) узкий изолированный пик при $\omega \sim 3100 \mathrm{~cm}^{-1}$; 2) большая группа близко расположенных максимумов, два из которых в 3-5 раз выше остальных, при $\omega=1000-1400 \mathrm{~cm}^{-1}$; 3) несколько близких максимумов, один из которых в $\sim 2$ раза больше других, 

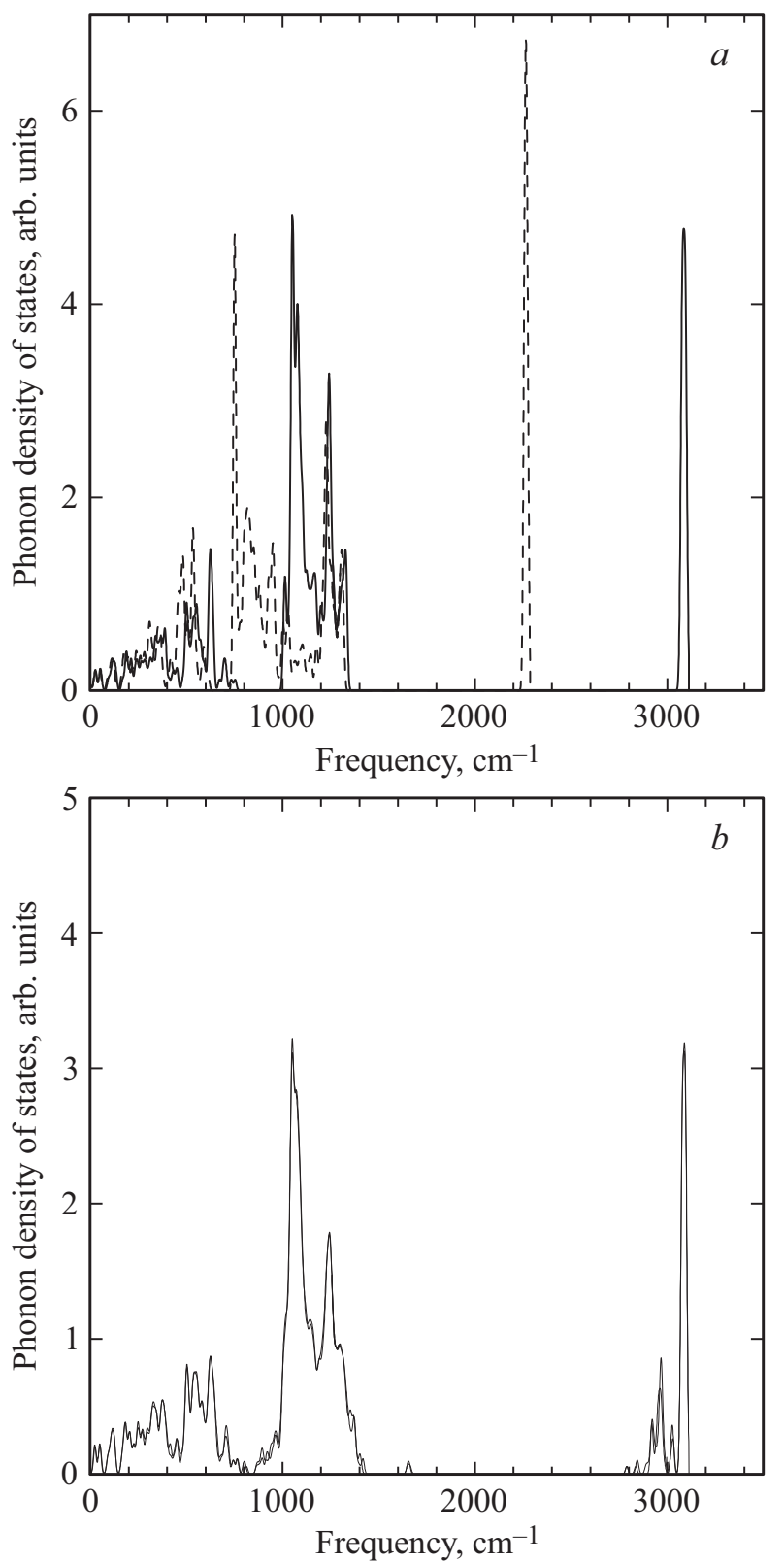

Рис. 2. $a-$ плотность фононных состояний графана до (сплошная линия) и после (штриховая линия) замены всех атомов водорода на атомы дейтерия (данные для сверхъячеек $\mathrm{C}_{160} \mathrm{H}_{160}$ и $\mathrm{C}_{160} \mathrm{D}_{160}$ соответственно). $b$ - плотность фононных состояний сверхъячейки $\mathrm{C}_{160} \mathrm{H}_{144}$, соответствующей графану с $10 \%$ водородных вакансий.

при $\omega=500-700 \mathrm{~cm}^{-1}$ и 4) область без четко выраженных особенностей PDOS, в которой PDOS сравнительно мала и увеличивается с ростом $\omega$, при $\omega<400 \mathrm{~cm}^{-1}$.

Чтобы получить наглядное представление о том, колебания каких атомов дают вклад в PDOS на различных участках спектра, мы рассчитали PDOS сверхъячейки $\mathrm{C}_{160} \mathrm{D}_{160}$, в которой все атомы водорода заменены на атомы дейтерия (см. рис. 2,a). Из сравнения этой PDOS c PDOS графана видно, что высокочастотный пик обязан своим происхождением вибрациям атомов водорода в связях $\mathrm{C}-\mathrm{H}$, низкочастотная область спектра обусловлена преимущественно колебаниями углеродного остова, а в промежуточном диапазоне частот вклад в PDOS дают как углеродные, так и водородные моды с преобладанием последних.

Десорбция 10\% водорода приводит к уменьшению высоты максимумов PDOS при $\omega \sim 1050,1250$ и $3100 \mathrm{~cm}^{-1}$ на $\sim 35,45$ и $35 \%$ соответственно (см. рис. $2, b$ ). При дальнейшей десорбции эти максимумы продолжают уменьшаться. В сверхъячейке $\mathrm{C}_{160} \mathrm{H}_{80}$ с $50 \%$ водородных вакансий высокочастотный пик отсутствует, а два других максимума очень сильно размыты.

\section{2. Механическая жесткость и коэффициент Пуассона}

Механическая жесткость квазидвумерных материалов при одноосной деформации определяется как [22]

$$
Y^{\prime}=\frac{1}{S}\left(\frac{\partial^{2} E}{\partial \varepsilon^{2}}\right)_{\varepsilon=0},
$$

где $S$ - площадь образца, $E-$ его полная упругая энергия, $\varepsilon-$ относительная деформация в направлении действия растягивающей силы. Величина $Y^{\prime}$ измеряется в Н/м и связана с модулем Юнга $Y$ соотношением $Y=Y^{\prime} / d$, где $d-$ толщина образца. При численных расчетах выражение (1) удобно представить в виде

$$
Y^{\prime}=\frac{2 \Delta E}{S \varepsilon^{2}}
$$

где $\Delta E-$ приращение энергии при деформации, $\varepsilon \ll 1$ (малость этой величины необходима для гармонической реакции кристалла; в настоящей работе мы во всех расчетах полагали $\left.\varepsilon=10^{-3}\right)$. Если материал анизотропен в плоскости $(x, y)$, то можно говорить о его жесткости $Y_{x}^{\prime}$ и $Y_{y}^{\prime}$ в направлениях $x$ и $y$ соответственно (в изотропной среде $\left.Y_{x}^{\prime}=Y_{y}^{\prime} \equiv Y^{\prime}\right)$.

Для проверки нашего вычислительного алгоритма мы рассчитали жесткость графена, используя для этой цели сверхъячейку $\mathrm{C}_{160}$. Полученное нами значение $Y^{\prime}=324 \mathrm{H} /$ м согласуется с экспериментальной величиной $Y^{\prime}=340 \pm 50 \mathrm{H} / \mathrm{M}$ [27] и близко к имеющимся в литературе теоретическим данным других авторов $Y^{\prime}=335-353 \mathrm{H} / \mathrm{M}[8,10,17-19,28]$.

Для графановой сверхъячейки $\mathrm{C}_{160} \mathrm{H}_{160}$ мы получили $Y^{\prime}=249 \mathrm{H} /$ м - значительно меньше, чем в графене. Найденная нами величина не существенно отличается от приводимых в литературе значений $Y^{\prime}=224-246$ Н/м [8,10,17-19,28]. При вычислении модуля Юнга графена толщину монослоя графена принимают равной расстоянию между соседними графеновыми слоями в графите $d=3.35 \AA$ [22]. Для удобства сопоставления с графеном толщину слоя графана будем также полагать равной $d=3.35 \AA$. Тогда для модуля Юнга графана получим $Y=0.74$ ТПа (в графене $Y \sim 1 \mathrm{TПа).}$ 
Упругие модули графана с различным содержанием водорода. Результаты для сверхъячейки $\mathrm{C}_{160} \mathrm{H}_{x}$

\begin{tabular}{c|c|c}
\hline $\begin{array}{c}\text { Концентрация } \\
\text { водородных вакансий }\end{array}$ & $\begin{array}{c}\text { Механическая } \\
\text { жесткость, Н/м }\end{array}$ & $\begin{array}{c}\text { Коэффициент } \\
\text { Пуассона }\end{array}$ \\
\hline $0 \%$ (графан) & 249 & 0.23 \\
$0.6 \%$ (1 вакансия) & 245 & 0.22 \\
$10 \%$ & $215 \pm 12$ & $0.18 \pm 0.01$ \\
$30 \%$ & $169 \pm 18$ & $0.12 \pm 0.02$ \\
$50 \%$ & $134 \pm 11$ & $0.25 \pm 0.02$ \\
$75 \%$ & $168 \pm 26$ & $0.16 \pm 0.04$ \\
$100 \%$ (графен) & 324 & 0.35
\end{tabular}

Удаление одного атома водорода из сверхъячейки $\mathrm{C}_{160} \mathrm{H}_{160}$ (что соответствует десорбции $\sim 0.6 \%$ водорода из графана) приводит к уменьшению $Y^{\prime}$ до $245 \mathrm{H} / \mathrm{M}$. При дальнейшей десорбции величины $Y_{x}^{\prime}$ и $Y_{y}^{\prime}$ становятся очень чувствительными к конкретной конфигурации водородных вакансий, причем для данной конфигурации они могут весьма существенно отличаться друг от друга. По этой причине мы не вычисляем средние значения $Y_{x}^{\prime}$ и $Y_{y}^{\prime}$ по отдельности, а для каждой концентрации вакансий находим жесткость $Y^{\prime}$ как среднее не только по различным конфигурациям вакансий, но и по двум направлениям ( $x$ и $y)$. Полученные результаты представлены в таблице.

Видно, что с ростом концентрации водородных вакансий величина $Y^{\prime}$ сначала уменьшается, проходит через минимум $Y^{\prime} \sim 130 \mathrm{H} /$ м при десорбции $\sim 50 \%$ атомов водорода, а затем вновь увеличивается, достигая своего максимума в графене. Такое поведение механической жесткости при дегидрировании графана определяется двумя конкурирующими факторами: 1) уменьшением средней по образцу прочности связей $\mathrm{C}-\mathrm{C}$ из-за беспорядка, привносимого хаотически расположенными водородными вакансиями, и 2) постепенным увеличением средней прочности этих связей по мере перехода от графана к графену, в котором энергия одной связи $\mathrm{C}-\mathrm{C}$ больше, чем в графане.

Для квазидвумерных материалов коэффициент Пуассона в направлении $y$ при деформации в направлении $x$ вычисляется как [29,30]

$$
v_{y x}=-\varepsilon_{y} / \varepsilon_{x}
$$

где $\varepsilon_{x}$ и $\varepsilon_{y}$ - относительные деформации в направлениях $x$ и $y$ соответственно, причем величина $\varepsilon_{x}$ задается, a $\varepsilon_{y}$ определяется из условия минимума энергии образца. Аналогичный вид имеет формула для коэффициента Пуассона в направлении $x$ при растяжении вдоль $y$ :

$$
v_{x y}=-\varepsilon_{x} / \varepsilon_{y}
$$

за тем отличием, что теперь $\varepsilon_{y}$ является входным параметром, а $\varepsilon_{x}$ - выходным. В нашем случае роль длины и ширины образца играли периоды прямоугольной сверхъячейки.
В изотропных материалах (графен и графан практически изотропны) $v_{x y}=v_{y x}=v$. Для графена разброс имеющихся в литературе расчетных значений $v$ очень велик: $v=0.15-0.45$ (см. работу [22] и ссылки в ней). Найденная нами величина $v=0.35$ в графене [30] согласуется с результатом работы [31].

Для графановой сверхъячейки $\mathrm{C}_{160} \mathrm{H}_{160}$ мы получили $v=0.23$. Это существенно больше приводимых в литературе теоретических значений $v=0.07-0.09$ в графане [8,10,16,17-19]. Причина столь значительного несоответствия может заключаться, например, в использовании разных расчетных методик, различном типе граничных условий, разном числе атомов в модельном образце и т.д. Здесь нелишне напомнить о сильном разбросе теоретической величины $v$ в графене [22].

Как бы то ни было, мы изучили характер изменения $v$ при уменьшении содержания водорода в графане, используя для этой цели сверхъячейки $\mathrm{C}_{160} \mathrm{H}_{x}$ с различными $x$. Как и при расчете механической жесткости, для каждой концентрации водородных вакансий мы приводим величину $v$, полученную усреднением по различным конфигурациям вакансий и направлениям $x, y$ (см. таблицу).

Анализ полученных результатов показывает, что с ростом числа водородных вакансий коэффициент Пуассона осциллирует: сначала он уменьшается до минимального значения при концентрации вакансий $~ 30 \%$, затем проходит через локальный максимум и неглубокий минимум при десорбции $\sim 50$ и $\sim 75 \%$ водорода соответственно, после чего увеличивается до своей максимальной величины в графене. Обращает на себя внимание сравнительно большой разброс значений $v$ при фиксированном содержании водорода, что связано с чувствительностью $v$ к расположению водородных вакансий.

Мы не обнаружили наблюдавшегося в работе [16] минимума $v$ при половинном гидрировании графена. Это может быть связано как с недостаточно большими размерами использованной в настоящей работе сверхъячейки (и с флуктуациями плотности распределения водорода), так и с какими-то другими, не вполне понятными для нас причинами. Здесь требуются дополнительные исследования.

\section{3. Электронная структура}

Плотность электронных состояний сверхъячейки $\mathrm{C}_{160} \mathrm{H}_{160}$ приведена на рис. $3, a$. Видно, что графан является диэлектриком. Ширина запрещенной зоны $E_{g}$, определяемая как разность энергий нижней незаполненной (LUMO) и верхней заполненной (HOMO) молекулярной орбитали, составляет 5.34 эВ - в прекрасном соответствии с величиной $E_{g}=5.4$ эВ, полученной путем более строгих расчетов [32].

Десорбция одного атома водорода приводит к появлению вблизи дна запрещенной зоны локального уровня энергии (рис. 3,a). По мере десорбции число 

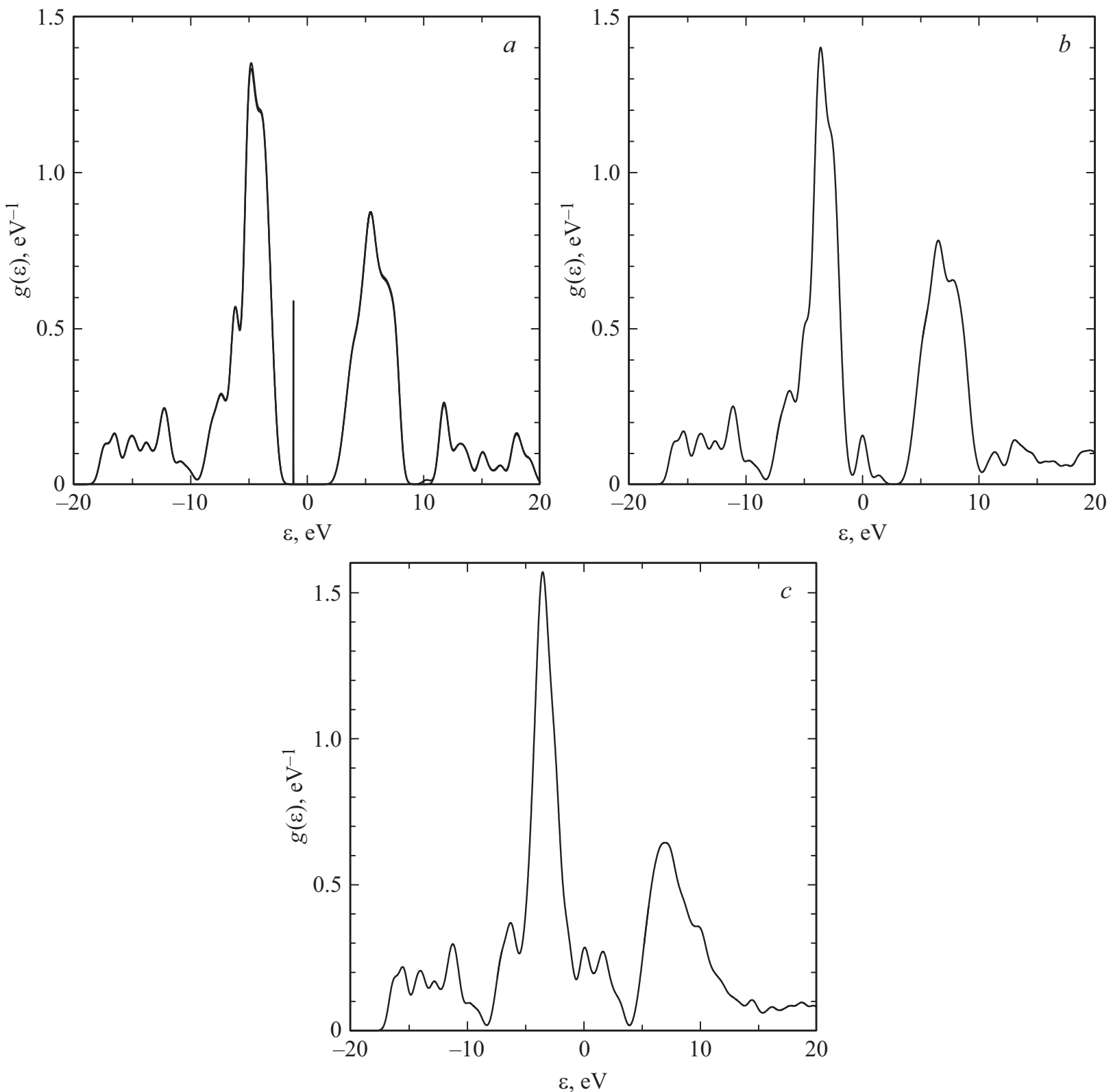

Рис. 3. Плотность электронных состояний $g(\varepsilon)$ в расчете на спин и на элементарную ячейку (сплошная линия) для сверхъячеек $\mathrm{C}_{160} \mathrm{H}_{160}(a), \mathrm{C}_{160} \mathrm{H}_{144}(b)$ и $\mathrm{C}_{160} \mathrm{H}_{80}(c)$. Тонкой вертикальной линией на $(a)$ показан локальный энергетический уровень, возникающий в сверхъячейке $\mathrm{C}_{160} \mathrm{H}_{159}$ при десорбции одного атома водорода. За начало отсчета энергии принят уровень Ферми.

таких уровней увеличивается. После десорбции 10\% водорода формируется примесная зона, в которой находится уровень Ферми (рис. $3, b$ ). При дальнейшей десорбции ширина этой зоны увеличивается, а плотность электронных состояний в ней может иметь несколько четко выраженных максимумов (рис. 3,c). При этом плотность электронных состояний на уровне Ферми всегда отлична от нуля, т.е. частично дегидрированный графан при низких температурах должен быть проводником. Полная десорбция водорода переводит графан в графен с характерной для него $V$-образной зависимостью плотности состояний от энергии (см. также [13]).

\section{4. Заключение}

1. Механическая жесткость графана значительно меньше жесткости графена. С ростом числа водородных вакансий она сначала уменьшается, проходит через минимум при десорбции $\sim 50 \%$ водорода, а затем вновь увеличивается.

2. Коэффициент Пуассона очень чувствителен к конкретной конфигурации водородных вакансий. Он минимален при десорбции $\sim 30 \%$ водорода.

3. Десорбция $\sim 10 \%$ водорода приводит к уменьшению максимумов плотности фононных состояний при $\sim 1050,1250$ и $3100 \mathrm{~cm}^{-1}$ на $\sim 35-45 \%$. 
4. По мере десорбции водорода в запрещенной энергетической зоне сначала возникают изолированные уровни, а затем формируется примесная зона, в которой находится уровень Ферми.

Работа выполнена при поддержке Министерства образования и науки РФ в рамках Программы повышения конкурентоспособности НИЯУ МИФИ. Работа поддержана грантом РФФИ № 15-02-02764.

\section{Список литературы}

[1] K.S. Novoselov, A.K. Geim, S.V. Morozov, D. Jiang, Y. Zhang, S.V. Dubonos, I.V. Grigorieva, A.A. Firsov. Science, 306, 666 (2004).

[2] A.K. Geim, K.S. Novoselov. Nature Mater., 6, 183 (2007).

[5] Л.А. Чернозатонский, П.Б. Сорокин, Е.Э. Белова, Й. Брюнинг, А.С. Федоров. Письма ЖЭТФ, 85, 84 (2007).

[3] Y.-W. Son, M.L. Cohen, S.G. Louie. Phys. Rev. Lett., 97, 216803 (2006)

[4] M.Y. Han, B. Özyilmaz, Y. Zhang, P. Kim. Phys. Rev. Lett., 98, 206805 (2007).

[6] J.O. Sofo, A.S. Chaudhari, G.D. Barber. Phys. Rev. B, 75, 153401 (2007).

[7] D.C. Elias, R.R. Nair, T.M.G. Mohiuddin, S.V. Morozov, P. Blake, M.P. Halsall, A.C. Ferrari, D.W. Boukhvalov, M.I. Katsnelson, A.K. Geim, K.S. Novoselov. Science, 323, 610 (2009).

[8] H. Sahin, O. Leenaerts, S.K. Singh, F.M. Peeters. WIREs Comput. Mol. Sci., 5, 255 (2015).

[9] Т.Е. Беленкова, В.М. Чернов, Е.А. Беленков. РЭНСИТ, 8, 49 (2016).

[10] E. Cadelano, P.L. Palla, S. Giordano, L. Colombo. Phys. Rev. B, 82, 235414 (2010).

[11] R.E. Mapasha, M.P. Molepo, N. Chetty. Physica E, 79, 52 (2016).

[12] H.Sahin, C. Ataca, S. Ciraci. Appl. Phys. Lett., 95, 222510 (1009).

[13] P. Chandrachud, B. Pujari, S. Halder, B. Sanyal, D.G. Kanhere. J. Phys.: Condens. Matter, 22, 465502 (2010).

[14] Л.А. Опенов, А.И. Подливаев. Письма ЖЭТФ, 90, 505 (2009).

[15] C.D. Reddy, Y.-W. Zhang. Carbon, 69, 86 (2014).

[16] J.W. Jiang, T. Chang, X. Guo. Nanoscale, 8, 15948 (2016).

[17] R. Ansari, M. Mirnezhad, H. Rouhi. Sol. St. Commun., 201, 1 (2015).

[18] O. Leenaerts, H. Peelaers, A.D. Hernandez-Nieves, B. Partoens, F.M. Peeters. Phys. Rev. B, 82, 195436 (2010).

[19] Topsacal, S. Cahangirov, S. Ciraci. Appl. Phys. Lett., 96, 091912 (2010).

[20] Л.А. Опенов, А.И. Подливаев. Письма ЖТФ, 36, 69 (2010).

[21] H.-C. Huang, S-Y. Lin, C.-L. Wu, M.-F. Lin. Carbon, 103, 84 (2016).

[22] C.D. Reddy, S. Rajendran, K.M. Liew. Nanotechnology, 17, 864 (2006).

[23] M.M. Maslov, A.I. Podlivaev, K.P. Katin. Molecular Simulation, 42, 305 (2016).

[24] А.И. Подливаев, Л.А. Опенов. ФТП, 51, 222 (2017).

[25] А.И. Подливаев, Л.А. Опенов. ФТП, 51, 667 (2017).

[26] H. Peelaers, A.D. Hernandez-Nieves, O. Leenaerts, B. Partoens, F.M. Peeters. Appl. Phys. Lett., 98, 051914 (2011).
[27] C. Lee, X. Wei, J.W. Kysar, J. Hone. Science, 321, 385 (2008).

[28] E. Munoz, A.K. Singh, M.A. Ribas, E.S. Penev, B.I. Yakobson. Diamond Rel. Mater., 19, 368 (2010).

[29] Б.Д. Аннин, Н.И. Остросаблин. Прикл. механика и пром. физика, 49, 131 (2008).

[30] Л.А. Опенов, А.И. Подливаев. ФТТ, 59, 1240 (2017).

[31] J.-W. Jiang, H.S. Park. Nano Lett., 16, 2657 (2016).

[32] S. Lebg̀ue, M. Klintenberg, O. Eriksson, M.I. Katsnelson. Rev. B, 79, 245117 (2009).

Редактор А.Н. Смирнов

\section{Effect of graphane dehydrogenation on its mechanical and electronic properties}

\section{L.A. Openov, A.I. Podlivaev} National Research Nuclear University „MePhil“,
115409 Moscow, Russia

Abstract The effect of hydrogen desorption on the mechanical characteristics and electronic structure of the chair conformation of graphane is studied in the context of the nonorthogonal tight binding model. In is shown that the stiffness and the Poisson ratio are nonmonotonic functions of the hydrogen content and have their minima at the concentration of hydrogen vacancies $\sim 50$ and $\sim 30 \%$ respectively. The characteristic peaks in the phonon density of states rapidly diminish upon hydrogen desorption. At the initial stage of desorption, local energy levels appear in the band gap. As the number of hydrogen vacancies increases, these levels form an impurity band in which the Fermi level is located. 